\title{
INCIDENCIA DE LA IMAGEN
} CORPORATIVA EN EL POSICIONAMIENTO DE LAS VENTAS, MEDIANTE UNA INVESTIGACIÓN DE MERCADO QUE INVOLUCRE A LOS CLIENTES.

Nathyn Collins Ventura, Adrián Valencia Medranda, Jairo Cedeño Pinoargote, Irene Palacios Bauz, Karina Santistevan Villacreses 


\title{
INCIDENCIA DE LA IMAGEN CORPORATIVA EN EL POSICIONAMIENTO DE LAS VENTAS, MEDIANTE UNA INVESTIGACIÓN DE MERCADO QUE INVOLUCRE A LOS CLIENTES.
}

\author{
Nathyn Collins Ventura ${ }^{1}$, Adrián Valencia Medranda ${ }^{1}$, Jairo Cedeño Pinoargote ${ }^{1}$, Irene Palacios Bauz ${ }^{1}$, \\ Karina Lourdes Santistevan Villacreses ${ }^{1}$. \\ ${ }^{1}$ Universidad Estatal Península de Santa Elena. Carrera de Marketing. \\ Campus La Libertad, vía principal Santa Elena - La Libertad - Ecuador \\ E-mail: collinsnat_17@live.com
}

\begin{abstract}
RESUMEN
En la actualidad las empresas necesitan crear una Imagen idónea, compuesta por elementos identificadores distintivos, que permitan la diferenciación por parte de la empresa y a la vez genera atención, aceptación y confianza al cliente. Así mismo, parte de esta Imagen, es lograr el posicionamiento positivo de la empresa en el cliente, esto consiste en ocupar un lugar en la mente y corazón del consumidor, para que en el proceso de la compra, sea este un signo distintivo que permita al final elegir el producto que oferta aquella empresa. En este trabajo se desarrolla a partir de una investigación de campo y bibliográfica que analiza profundamente encuesta y sesiones de grupo la influencia de la imagen corporativa y posicionamiento en las ventas. Los datos del estudio muestran el alto nivel de influencia que se origina por una imagen acorde a las expectativas de los clientes, las cuales permiten crear estima y relevancia de marca de una empresa ante el cliente meta.
\end{abstract}

Palabras Claves: Imagen corporativa, Posicionamiento, Estrategias.

\begin{abstract}
Today companies need to create an ideal image, composed of distinctive identifiers that allow differentiation by the company while generating attention, customer acceptance and trust. Also, part of this picture is to achieve positive positioning of the company in the customer, this is a place in the hearts and minds of the consumer, so that in the process of purchase, this is a distinctive sign which allows the end choose the product that this company offer. This paper analyzes from a survey and focus groups the influence of corporate image and sales positioning. The study data show the high level of influence that arises from an image according to the expectations of customers, which allow you to create estimates and significance of a company's brand to the target customer.
\end{abstract}

Keywords: Corporate image, Positioning, Strategies. 


\section{Introducción.}

En la actualidad la imagen corporativa adquiere una importancia fundamental, el cual consiste en crear valor para la empresa y ser parte del activo intangible estratégico de la misma (Limón Peña, 2008), Ante la apertura de los mercados, y la creciente globalización, las empresas necesitan elementos identificadores distintivos, estos permiten la diferenciación por parte de la empresa y a la vez genera atención, aceptación y confianza al cliente.

\subsection{Objetivo de la imagen corporativa en una organización.}

(Kotler \& Amstrong, 2013) La Imagen Corporativa, se refiere a como se percibe una organización, es decir lo que esta significa, debe ser atractiva para provocar el interés al público, clientes, usuarios, consumidores. Así mismo, parte de esta Imagen, es lograr el posicionamiento de la empresa en el cliente, esto consiste en ocupar un lugar en la mente y corazón del consumidor, para que en el proceso de la compra sea este un signo distintivo que permita al final elegir el producto que oferta aquella empresa. Aquel proceso, culmina con las ventas, esta es una disciplina tan antigua como el hombre. (ECHEVERRI CAÑAS, 2009). Las ventas, es un proceso organizado orientado a potenciar la relación vendedorcliente, con el fin de persuadirle para obtener los productos de la empresa.

\subsection{Elementos identificadores distintivos de una empresa.}

(Limón Peña, 2008) Las empresas necesitan elementos identificadores distintivos, estos permiten la diferenciación por parte de la empresa y a la vez genera atención, aceptación y confianza al cliente. Así mismo, parte de esta Imagen, es lograr el posicionamiento de la empresa en el cliente, esto consiste en ocupa un lugar en la mente y corazón del consumidor, para que en el proceso de la compra, sea este un signo distintivo que permita al final elegir el producto que oferta aquella empresa (Stanton, Etzel, \& Walker, 2007). Las ventas son el corazón de cualquier negocio, es la actividad fundamental de aquella aventura comercial. Se trata de reunir a compradores y vendedores, y el trabajo de toda la organización es hacer lo necesario para que esta reunión sea exitosa.

\section{Materiales y métodos.}

Para el desarrollo de esta investigación, se empleó la técnica de encuestas como herramienta para analizar la forma en que incide la imagen corporativa y el posicionamiento en las ventas de una empresa. En esta investigación se utilizó el enfoque cuantitativo en donde se trabajó en base a una población y se obtuvo una muestra de 384 habitantes de la provincia de Santa Elena, tomando en cuenta las 3 jurisdicciones que encierra esta provincia.

Por otro laso se empleó Sesión de Grupos, se llevaron a cabo para entender las expectativas que tienen los consumidores potenciales respecto a la imagen y posicionamiento y su incidencia en las ventas, así como las perspectivas que tiene sobre su competencia directa e indirecta. Se pidió a 36 personas de la provincia de Santa Elena, entre ellas 12 por cada ciudad, es decir, Santa Elena, La Libertad y Salinas, entre ellos la mitad eran de género masculino y la mitad femenino. Se realizó investigación en base a la empresa aromas y recuerdos, la misma que comercializa perfumes y recuerdos de excelente calidad a precios altos, ubicada en el centro comercial Paseo Shopping.

\subsection{Procesamiento y análisis.}

Para la investigación, se escogió al 64,9\%de la población de Santa Elena, la misma que está dentro del perfil de consumidor del local Aromas y Recuerdos (Inec, 2010).

Tabla No.- 1 Rango de Edades

\begin{tabular}{|l|r|r|}
\hline Rango de edad & 2010 & $\%$ \\
\hline De 95 y más ańos & 190 & $0,1 \%$ \\
\hline De 90 a 94 ańos & 521 & $0,2 \%$ \\
\hline De 85 a 89 años & 1.026 & $0,3 \%$ \\
\hline De 80 a 84 ańos & 2.012 & $0,7 \%$ \\
\hline De 75 a 79 ańos & 3.057 & $1,0 \%$ \\
\hline De 70 a 74 ańos & 4.344 & $1,4 \%$ \\
\hline De 65 a 69 años & 5.831 & $1,9 \%$ \\
\hline De 60 a 64 ańos & 7.149 & $2,3 \%$ \\
\hline De 55 a 59 ańos & 9.957 & $3,2 \%$ \\
\hline De 50 a 54 ańos & 12.010 & $3,9 \%$ \\
\hline De 45 a 49 ańos & 15.280 & $4,9 \%$ \\
\hline De 40 a 44 ańos & 17.237 & $5,6 \%$ \\
\hline De 35 a 39 ańos & 20.091 & $6,5 \%$ \\
\hline De 30 a 34 ańos & 22.967 & $7,4 \%$ \\
\hline De 25 a 29 ańos & 25.512 & $8,3 \%$ \\
\hline De 20 a 24 ańos & 27.175 & $8,8 \%$ \\
\hline De 15 a 19 ańos & 29.874 & $9,7 \%$ \\
\hline De 10 a 14 ańos & 33.446 & $10,8 \%$ \\
\hline De 5 a 9 ańos & 34.252 & $11,1 \%$ \\
\hline De 0 a 4 ańos & 36.762 & $11,9 \%$ \\
\hline Total & 308.693 & $10,0 \%$ \\
\hline \multicolumn{1}{|c|}{ Fuente: (Inec, 2010$)$} & \\
\hline
\end{tabular}

Para calcular el tamaño muestral de los consumidores se aplicó la fórmula muestras finitas (Bernal , 2010).

Ilustración No.- 1 Muestra Población Finita

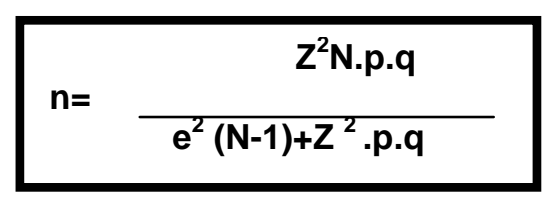


La siguiente tabla, indica el significado de los valores a utilizar.

Tabla No. 2. Significado de valores a utilizar en muestra

\begin{tabular}{|ccc|}
\hline \multicolumn{2}{|c|}{ Significado } & Valores \\
\hline N & Población o universo & 200.341 \\
\hline $\mathbf{n}$ & Tamaño de la muestra & $?$ \\
\hline $\mathbf{Z}$ & Nivel de confianza (95\%) & 1.96 \\
\hline $\mathbf{P}$ & Aceptación & 0.5 \\
\hline $\mathbf{q}$ & No aceptación & 0.5 \\
\hline $\mathbf{e}$ & Margen de error & 0.05 \\
\hline
\end{tabular}

Sustituyendo los valores:

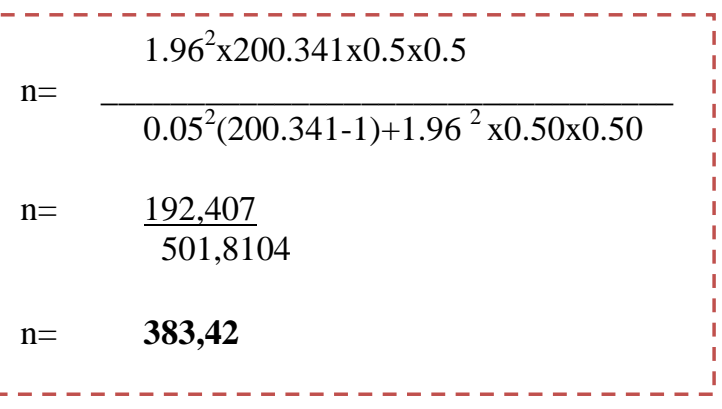

El número de personas encuestada fue 382 personas.

La información se procesó en función de los instrumentos y técnicas utilizadas, en la investigación de los perfiles de los consumidores de la Península de Santa Elena. En lo que se refiere a la tabulación, se utilizó el programa estadístico SPSS 10.1.

\section{Resultados.}

\subsection{Percepciones de la imagen corporativa ante la marca Aromas y Recuerdos.}

La percepción que tienen los clientes ante la marca "Aromas y Recuerdos", en su mayoría es de perfumes de excelente calidad, así mismo perciben la perciben como elegante que ofrece variedad de productos.

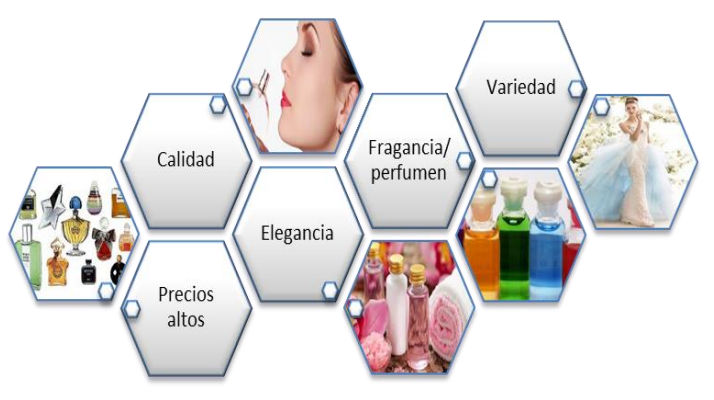

Gráfico No. 1. Percepción de los clientes ante la marca "Aromas y Recuerdos"

\subsection{Gestión de comunicación de la perfumería Aromas y Recuerdos.}

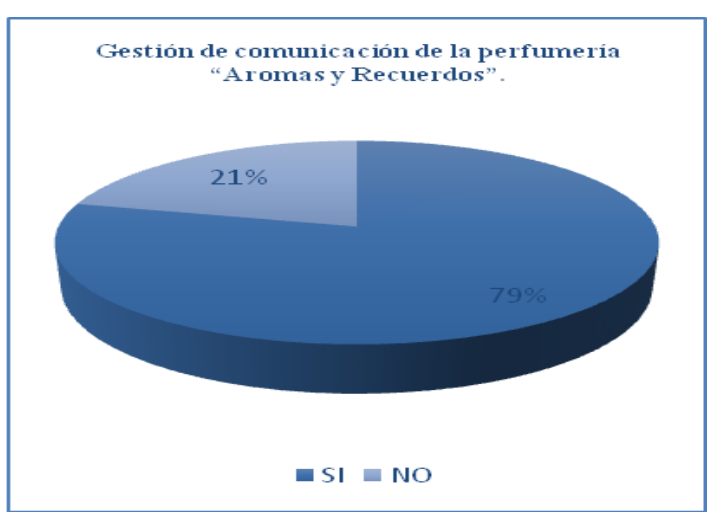

Gráfico No. 2. Gestión de comunicación de la perfumería "Aromas y Recuerdos".

En su mayoría conocen sobre la marca "Aromas y Recuerdos", lo cual se ve reflejado por la aceptación que tiene la empresa a nivel local. Por ello es de gran importancia, gestionar la imagen comunicativamente a través de la comunicación interna y la comunicación corporativa, queda excluida la comunicación del producto (publicitaria), considerado por Limón Peña (2008).

\subsection{Estímulos y sensaciones causadas por la imagen de perfumería Aromas y Recuerdos.}

Los estímulos y sensaciones causadas por la imagen de "Aromas y Recuerdos", son buenos en su mayoría. La calidad de la sensación provocada por una empresa es lo que permite diferenciarla de otra. Por ende debe gestionarse adecuadamente acciones que permitan generar estímulos favorables en cuanto a la imagen corporativa de una empresa. 


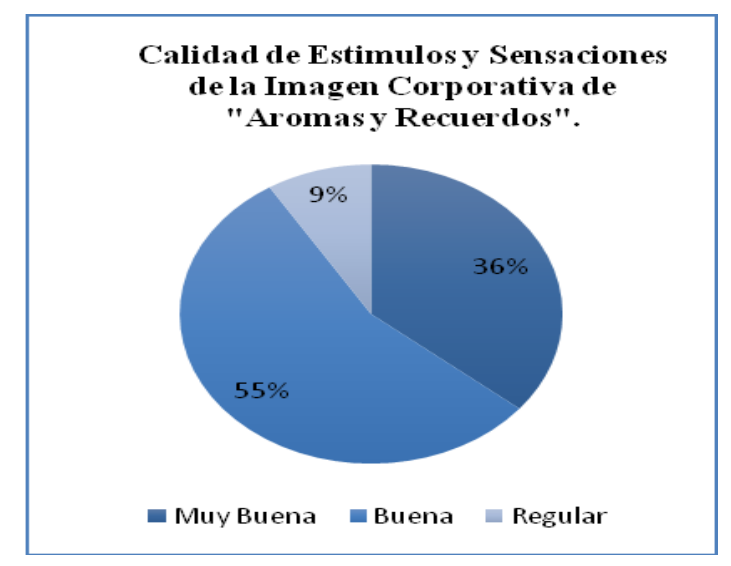

Gráfico No. 3 Calidad de Estímulos y sensaciones de la imagen corporativa de "Aromas y Recuerdos".

3.4 Representación mental de la identidad corporativa de Aromas $y$ Recuerdos

Mediante la Sesión de Grupo realizada se determinó que la representación mental, y concepto que tiene un público acerca de la perfumería son: Que es una Chica de 24 años de edad, hija única, con un nivel de estudio Superior empezando su Doctorado, una posición económica alta, con buena presencia y amante al diseño gráfico y por ende la moda, le gusta coleccionar perfumes, se distingue por ser elegante y realza mucho su belleza con glamour, su defecto es ser muy sensible, es de otro país y le fascina estar en el Paseo Shopping.

\subsection{Análisis de la imagen corporativa de los competidores potenciales.}

En comparación con aromas y recuerdos, Yambal es considerada de clase alta pero no con estudios superiores, mujer de 50 años, mientras las demás marcas son juveniles es decir de 20 a 26 años, nivel socioeconómico medio y son ecuatorianas.

\subsection{Identidad sectorial de la perfumería Aromas y Recuerdos en relación a los competidores potenciales.}

En relación a los competidores potenciales, la perfumería "Aromas y Recuerdos", se diferencia por los precios altos, debido a la alta calidad de los productos que se ofrece, acompañado de un empaque y envoltura llamativos y atractivos.
Tabla No. 3 Identidad sectorial de la perfumería "Aromas y Recuerdos".

\begin{tabular}{|c|c|c|c|c|}
$\begin{array}{c}\text { MARCA/ } \\
\text { CARACTERi } \\
\text { STICA }\end{array}$ & $\begin{array}{c}\text { Precios } \\
\text { Altos }\end{array}$ & Variedad & Calidad & $\begin{array}{c}\text { Empaque y } \\
\text { Envoltura }\end{array}$ \\
\hline $\begin{array}{c}\text { Aromas y } \\
\text { Recuerdos }\end{array}$ & 26,6 & 12,8 & 22,4 & 15 \\
\hline Yanbal & 25,5 & 18,5 & 17 & 14,5 \\
\hline Avon & 16 & 16,2 & 12,9 & 14 \\
\hline Oriflame & 22,2 & 14 & 13,7 & 14 \\
\hline Esika & 0 & 10,5 & 8,3 & 14 \\
\hline L'bel & 4,4 & 13 & 15,3 & 14,5 \\
\hline Cyzone & 5,3 & 15 & 10,4 & 14 \\
\hline TOTALES & 100 & 100 & 100 & 100 \\
\hline
\end{tabular}

\section{Conclusiones.}

- La percepción de algunas personas conocen la marca "Aromas y Recuerdos" es de costos elevados, teniendo como consecuencia el no poder adquirir de inmediato un producto de aquella marca y sustituirlo con el de los competidores potenciales.

- Las empresas deben considerar de gran importancia el diferenciar los estímulos externos provocados por la imagen corporativa, para convertirlos en conceptos que permitan generar sensaciones idóneas dirigidas a los clientes.

- La adecuada gestión de la imagen corporativa proyectada a un público determinado permite crear empatía entre ambos.

- La calidad de la sensación provocada por una empresa es lo que permite diferenciarla de otra. Por ende debe gestionarse adecuadamente acciones que permitan generar estímulos favorables en cuanto a la imagen corporativa de una empresa.

\section{Agradecimiento.}

Mi más sincero agradecimiento al Ing. Jimmy Candell Soto, Rector de la muy ilustre Universidad Estatal Península de Santa Elena por su arduo trabajo y por gestionar el aporte de la comunidad universitaria en difusión de artículos formativos de gran interés; a todas las personas que colaboraron para que esta investigación saliera de la mejor manera posible. 


\section{Bibliografía.}

1. Bernal, C. A. (2010). Metodología de la Investigación (Tercera Edición ed.). (O. Fernández Palma, Ed.) Bogotá, Colombia: Pearson Education.

2. Echeverri cañas, 1. M. (2009). Marketing práctico. Madrid, España: starbook.

3. Inec. (2010). Inec Santa Elena. Santa Elena.

4. Kotler, P., \& Amstrong, G. (2013). Fundamentos de Marketing (Decima primera Edición ed.). (G. Domínguez Chávez, Ed.) Naucalpan de Juárez, México: Pearson Educacion.

5. Limón Peña, M. (2008). Imagen corporativa: estrategia organizacional de comunicación global. Trillas: Editorial Trillas.

6. Stanton, W. J., Etzel, M. J., \& Walker, B. J. (2007). Fundamentos de Marketing. México, D.F.: McGRAW-HILL. 